$\angle$ Research Square

\title{
Management of button batteries in the upper gastrointestinal tract of children
}

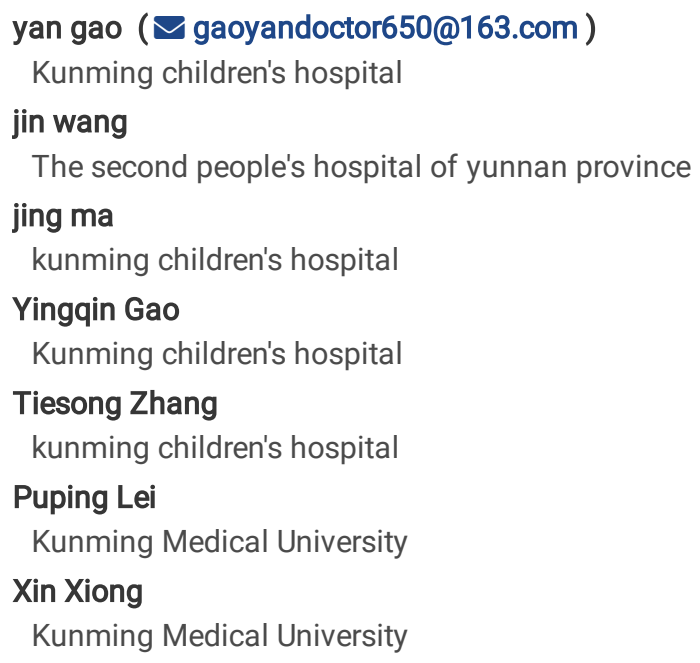

Keywords: button battery, children, esophageal stenosis

Posted Date: October 2nd, 2019

DOI: https://doi.org/10.21203/rs.2.15428/v1

License: (c) (1) This work is licensed under a Creative Commons Attribution 4.0 International License. Read Full License 


\section{Abstract}

Background: To analyze the lodging locations of ingested button batteries in the upper gastrointestinal tract of children, common complications and treatment effects.

Methods: The clinical data of 14 children with ingested button batteries lodged in the upper gastrointestinal tract were analyzed in our hospital between 2017 and 2019.

Results: Among 10 children with ingested button batteries lodged in the first stenosis of esophagus, 9 were cured and 1 suffered from esophagotracheal fistula 12 days after removal. One child with the ingested button battery lodged in the second stenosis of esophagus was dead due to intercurrent aortoesophageal fistula 13 days after removal. Two cases had ingested button batteries lodged in the third stenosis of esophagus, and were cured after removal. The ingested button battery in 1 case was lodged in the gastrointestinal tract, and discharged spontaneously 1 day after hospital admission.

Conclusions: Ingested button batteries are mainly lodged in the esophageal stenoses. Longer retention time could induce esophageal fistula. The ingested button battery in the gastrointestinal tract can discharge spontaneously.

\section{Background}

Ingested gastrointestinal foreign bodies are common in the otorhinolaryngology department of children, more prevalent in children less than 5 years old [1, 2]. With the development of electronic products, the number of children ingesting button batteries increases [3]. The leakage of alkaline content in button batteries can severely damage the gastrointestinal mucosae, and may lead to severe complications. However, the studies on the management of ingested button batteries in children are relatively rare. Herein, the clinical data of 14 children with ingested button batteries in the upper gastrointestinal tract were retrospectively analyzed in this study.

\section{Methods}

\section{Diagnosis and management}

The diagnosis was made primarily by the history of ingested button batteries, physical examination and chest-abdomen X-ray examination. The ingested button batteries lodged in the esophagus were removed by esophagoscope under general anesthesia in emergency room. The children with button batteries in the gastrointestinal tract were under observation, the removal of button batteries under an esophagoscope or open surgery would be conducted if necessary.

\section{Operation procedures}

The children in a supine nutation position underwent endotracheal intubation for general anesthesia. Esophagoscopy: various models of Germany Storz children's hard esophagoscopes, model number: $10 \mathrm{~mm} \times 12 \mathrm{~mm} \times 20 \mathrm{~mm}$ and $12 \mathrm{~mm} \times 14 \mathrm{~mm} \times 30 \mathrm{~mm}$; various models of Storz esophageal foreign body forceps and external Storz light source for illumination using a Mitsubishi mirror.

\section{Results}

\section{Basic information of children}

Among 14 children, there were 10 males and 4 females. Three cases (21.43\%) were at the age of 1 year, $9(64.29 \%)$ at the age of $1-4$ years and $2(14.29 \%)$ at the age of 5 years or older. The retention time of button batteries was $1-4 \mathrm{~h}$ in 2 cases $(14.29 \%), 4-24 \mathrm{~h}$ in 7 cases (50.00\%) and $>24 \mathrm{~h}$ in 5 cases (35.71\%), respectively. The number of children with the diameter of button batteries $<20 \mathrm{~mm},=20 \mathrm{~mm}$ and $>20 \mathrm{~mm}$ was 6 (42.86\%), 2 (14.29\%) and 6 (42.86\%), respectively. The occurred symptoms included fever (3 cases, $21.43 \%)$, dysphagia (12 cases, $85.71 \%)$, salivation (9 cases, $64.29 \%)$, vomiting (8 cases, $57.14 \%$ ), laryngeal stridor (1 case, $7.14 \%$ ) and abdominal pain ( 2 cases, $14.30 \%)$; only one case (7.14\%) was asymptomatic. The data of children was showed in Table 1.

\section{Lodging locations of ingested button batteries}

The ingested button batteries in 13 children were lodged in the esophagus (Fig. 1A-F). After hospital admission, the esophagoscopy was used to remove the button batteries under general anesthesia. The esophageal mucosa in 11 children were seriously erosive, the other two were just congestive and swollen. The battery exudation was wiped gently using cotton balls with distilled water. The ingested button batteries in 10 children were lodged in the first esophageal stenosis, with odynophagia, salivation, nausea and vomiting as major manifestations. Nine children were cured after removing button batteries. The other one was complicated with esophagotracheal fistula 4 days after removal (Fig. 2). One case with the ingested button battery lodged in the second esophageal stenosis was finally dead due to intercurrent aortoesophageal 
fistula 13 days after removal (Fig. 3). Two cases with the ingested button batteries lodged in the third esophageal esophagus were cured after removal. The ingested button battery in the last child stayed in the gastrointestinal tract, without any symptoms (Fig. 1G-H). The ingested button battery was discharged spontaneously in the following day. All the children were followed up until a normal diet or death.

The case complicated with esophagotracheal fistula: A 17-month-old child had the ingested button battery lodged in the first stenosis of esophagus. The chest radiography result showed a circular metal-density shadow at the level of C7-T1 (Fig. 1A-B). The retention time of button battery was 52 hours. A 22-mm-diameter lithium battery was taken out from esophagus. The child suffered from severe pneumonia on day 4 of postoperation and was diagnosed as esophagotracheal fistula (Fig. 2) through gastroscope examination on day 12 of postoperation (Given the pneumonia due to esophagotracheal fistula, we thought that esophagotracheal fistula occurred on day 4 of postoperation). Indwelling of the nasal feeding tube and parenteral nutrition were performed. The child was discharged from hospital with the nasal feeding tube after controlling pneumonia. As anticipated, esophagotracheal fistula was healed 136 days after removal and the nasal feeding tube was removed.

The death case: A 29-month-old child suffered from the ingested button battery lodged in the second stenosis of esophagus. The child was taken to hospital due to fever and obvious vomiting in the second day. Chest radiography result showed a circular high-density shadow at the level of T6-7 (Fig.1C-D). Preoperatively, the button battery remained in the body for 76 hours. A 21-mm-diameter lithium battery was taken out successfully using esophagoscope. The child was discharged from hospital with the symptom controlled. In the $13^{\text {th }}$ day after operation, the child suddenly spitted massive blood and was dead on the way of sending to the hospital. The autopsy result showed that the child died of hemorrhagic shock caused by severe bleeding due to the esophageal erosion and aortoesophageal fistula (Fig. 3).

The chief complaint of two children with ingested button batteries lodged in the third stenosis of esophagus was abdominal pain. The chest radiography results showed a high-density shadow at the level of T11 and presence of possible foreign bodies in the lower segment of esophagus (Fig. 1E-F). Two button batteries $(20 \mathrm{~mm}$ and $21 \mathrm{~mm}$ ) were taken out using esophagoscope. Even though the esophageal mucosae were edematous and erosive, esophageal and cardiac stenosis did not occur in following weeks.

The last child with the ingested button battery in the gastrointestinal tract was 8 and half years old. The thoracic and abdominal radiography results showed that a sheet of high-density shadow was present in the superior margin of L5 in the middle abdomen, and the edge was smooth (Fig. 1G-H). A 10-mm-button battery was discharged spontaneously within 24 hours. No gastrointestinal erosive signs and complications occurred through observation.

\section{Discussion}

Compared with other foreign bodies, such as nuts, rubber, scroll, plastic toys and coins, the button batteries ingested by children are prone to cause severe complications. The button batteries are characterized by metallicity, strong basicity $(\mathrm{pH}=13.5)$, strong corrosion, electrification and local compression [4], but seldomly lead to the increase of heavy metal content in blood and urine [5]. The diameter of batteries usually is 5.8-30 mm (alkaline or silver oxide batteries: $7-12 \mathrm{~mm}$; lithium batteries: $16-20 \mathrm{~mm}$ ) and thickness is $1.2-5.4 \mathrm{~mm}$, and generate voltages is 1.5-3 $\mathrm{V}$ based on different sizes [6]. With the increasing of battery diameters and extensive application of lithium batteries, the prevalence and mortality of button battery ingestion are on the rise [7]. From 1990 to 2008, the prevalence of lithium battery ingestion increased from 1.3\% to approximately $25 \%$. Meanwhile, the prevalence of button battery ingestion with the diameter of $20-25 \mathrm{~mm}$ increased from $1 \%$ to $18 \%$ [8].

The typical manifestations of children ingesting button batteries are divided into three types, including: (1) irritability, anorexia, tarry stool and dysphonia in children below 1 years old; (2) fever, dyspnea, cough, salivation and vomiting in children aged 1-4 years old; (3) abdominal pain and chest pain in children above 5 years old [9]. The imaging results showed that the ingested button battery was usually presented to be a typical dicyclic image in anteroposterior posture and a stagewise shadow in the lateral posture [10,11]. All the children ingesting button batteries are diagnosed through medical history and the imaging results. Button batteries tend to lodge in the stenosis of esophagus around which the trachea, mediastinum and great vessels exist. In addition, the damage of esophagus, as an important passage of feeding, can result in serious complications including spondylitis [12], esophageal perforation [13], esophageal stenosis, esophagotracheal fistula [14], aortoesophageal fistula[15], vocal cord paralysis[16] and Meckel diverticulum [17, 18].

In this study, 10 children (71.4\%) with ingested button batteries lodged in the first stenosis of esophagus had obvious symptoms, so they were early discovered and sent to the hospital timely. After hospital admission, 9 cases $(90.0 \%)$ were cured by positive treatment after removal. Although 1 case (10.0\%) was subjected to esophagotracheal fistula, he was cured completely by removing the ingested button battery and parenteral nutrition, indicating an extremely strong repair capability of children. Only 1 child (7.1\%) experienced the ingested button battery lodged in the second stenosis of esophagus, and was dead due to aortoesophageal fistula. Due to different anatomical positions, the first esophageal stenosis is easy to complicate with esophagotracheal fistula, while the second stenosis is easily complicated with aortoesophageal fistula. Thus, ingested button batteries lodged in the second esophageal stenosis should be given more positive prevention treatment to prevent aortoesophageal fistula. Two children (14.2\%) with ingested button batteries lodged in the third stenosis of esophagus were sent to hospital in time due to abdominal pain, leading to a good prognosis. 
The button battery is apt to cause a short circuit under the circumstance of moist esophagus, and then starts to electrolyze, discharge and release alkaline substances to damage the body. The window of opportunity for injury-free removal of an esophageal battery is <2 hours [8]. Additionally, these erosive and perforating effects may also continue even after removing button batteries [19, 20]. In this study, the retention time of all batteries was over 2 hours, and the esophageal mucosae were complicated with swelling, errhysis, hyperaemia and other symptoms. The retention time of 2 children with esophageal fistula was respectively $55 \mathrm{~h}$ and $76 \mathrm{~h}$, significantly longer than those without esophageal fistula (except for No. 6 child), suggesting that the battery retention time may be an important influencing factor for the development of esophageal fistula. Therefore, for the children with the retention time of ingested button batteries longer than $50 \mathrm{~h}$, the preventive management should be taken to prevent the occurrence of complications. Except for the battery retention time, the other factors, such as the battery lodging location, types, charged voltage, size, corrosive degree and the child's age, are also important.

The external electrolytic current generates hydroxide at the negative battery pole, so the anatomic position and orientation of a battery lodged in the esophagus may predict the specific subsequent injury [8]. Clinical expectations can be guided by the 3-Ns mnemonic "negative-narrownecrotic" theory: the negative battery pole, identifiable as the narrow side on lateral radiograph, causes the most severe necrotic injury. Intraoperatively, the distilled water was used to gently wipe the battery exudation on the mucosa. Postoperatively, nasogastric liquid diet or intravenous nutrition is proposed to protect esophageal mucosae. The study showed that tracheoesophageal fistulas became symptomatic within 9 days after removing batteries, and fistulation into an artery as late as 18 days after removal [8]. Our results suggested that tracheoesophageal fistula and aortoesophageal fistula occurred 4 and 13 days after removals, respectively. Given the lethality of aortoesophageal fistula, high-risk children with ingested button batteries lodged in the second stenosis of esophagus should remain in hospital under observation at least 18 days after removal.

According to the guidelines for the management of ingested foreign bodies in the gastrointestinal tract in 2002, it was unnecessary to take out the batteries below the esophagus unless the symptoms and signs of gastrointestinal injury appeared, or a large-diameter battery (>20 mm in diameter) remains in the stomach for more than $48 \mathrm{~h}$ by repeated X-ray examinations [21]. In 2015, North American Society for Pediatric Gastroenterology, Hepatology and Nutrition (NASPGHAN) Endoscope Committee also proposed that the children with the following factors, such as 5 years older or above, short duration of ingestion ( $<2$ hours), size of the battery $<20 \mathrm{~mm}$, absence of clinical symptoms, should be observed alone [22]. Various digestive juices and enzymes in gastric acid and gastrointestinal tract can neutralize alkaline substance released by the battery, which reduce the corrosive effect of batteries. Additionally, the button batteries passing beyond esophagus are uneasy to lodge in the gastrointestinal tract.

Prevention remains the best treatment for ingested button batteries [23]. On one hand, the manufacturing techniques or appearance of batteries and battery boxes should be improved and the executive standards for battery production should be strictly controlled. On the other hand, the button battery ingestion can be avoided by enhancing health propaganda, family education and safeguard. The children should be sent to the hospital as soon as possible once the battery is ingested. Furthermore, specialist physicians also need to participate in training. Early diagnosis, timely management and comprehensive treatment are conductive to a reduction of complications.

\section{Conclusions}

Ingested button batteries are mainly lodged in the esophageal stenoses. Longer retention time could induce esophageal fistula. The ingested button battery in the gastrointestinal tract can discharge spontaneously.

\section{Declarations}

Ethics approval and consent to participate

Written informed consent for participation in the study was obtained where participants are children (under 16 years old) from their parents or guardians and this study was approval from the Ethics Committee of Children's Hospital Affiliated to Kunming Medical

University(20190917001).

Consent for publication

\section{Not applicable.}

Availability of data and materials

\section{Not applicable.}


The authors declare that they have no competing interests.

Funding

\title{
Not applicable.
}

\author{
Authors' contributions
}

Y. G. and J. W. were responsible for designing the concept and writing the article; J. M., Y. G., T. Z., P. L.and X. X. participated in data collection and data analysis; Y. G. was involved in supervising the article. The final article was approved by all the authors.

Acknowledgement

\section{Not applicable.}

\section{References}

1. Rodríguez H, Passali GC, Gregori D, Chinski A, Tiscornia C, Botto H, et al. Management of foreign bodies in the airway and oesophagus. Int J Pediatr Otorhinolaryngol 2012;76(Suppl 1): S84-91. 2. Altokhais TI, Al-Saleem A, Gado A, Al-Qahtani A, Al-Bassam A. Esophageal foreign bodies in children: Emphasis on complicated cases. Asian J Surg 2017;40(5):362-366. 3. Lee JH, Lee JH, Shim JO, Lee JH, Eun BL, Yoo KH. Foreign Body Ingestion in Children: Should Button Batteries in the Stomach Be Urgently Removed? Pediatr Gastroenterol Hepatol Nutr 2016;19(1):20-28. 4. Farrow C. Button battery exposure clinical features and management. Emerg Nurse 2000;8(8):12-17. 5. Guinet T, Gaulier JM, Moesch C, Bagur J, Malicier D, Maujean G. Sudden death following accidental ingestion of a button battery by a 17-month-old child: a case study. Int J Legal Med 2016;130(5):1291-1297. 6. Labadie M, O'Mahony E, Capaldo L, Courtois A, Lamireau T, Nisse P, et al. Severity of button batteries ingestions: data from French Poison Control Centres between 1999 and 2015. Eur J Emerg Med 2018;25(4): e1-e8. 7. Bolton SM, Saker M, Bass LM. Button battery and magnet ingestions in the pediatric patient. Curr Opin Pediatr 2018;30(5):653-659. 8. Litovitz T, Whitaker N, Clark L, White NC, Marsolek M. Emerging battery-ingestion hazard: clinical implications. Pediatrics 2010;125(6):1168-1177. 9. Buttazzoni E, Gregori D, Paoli B, Soriani N, et al. Symptoms associated with button batteries injuries in children: An epidemiological review. Int J Pediatr Otorhinolaryngol 2015;79(12):2200-2207. 10. Jatana KR, Litovitz T, Reilly JS, Koltai PJ, Rider G, Jacobs IN. Pediatric button battery injuries: 2013 task force update. Int J Pediatr Otorhinolaryngol 2013;77(9):1392-1399. 11. Watanabe K, Hatano GY, Aoki H, Okubo K. The necessity of simple X-ray examination: a case report of button battery migration into the nasal cavity. Pediatr Emerg Care 2013;29(2):209-211. 12. Kieu V, Palit S, Wilson G, Ditchfield M, et al. Cervical spondylodiscitis following button battery ingestion. J Pediatr 2014;164(6):1500-1500.e1. 13. Gordon AC, Gough MH. Oesophageal perforation after button battery ingestion. Ann R Coll Surg Engl 1993;75(5):362-364. 14. Imamoğlu M, Cay A, Koşucu P, Ahmetoğlu A, Sarihan H. Acquired tracheo-esophageal fistulas caused by button battery lodged in the esophagus. Pediatr Surg Int 2004;20(4):292-294. 15. Brumbaugh DE, Colson SB, Sandoval JA, Karrer FM, Bealer JF, Litovitz T, et al. Management of button battery-induced hemorrhage in children. J Pediatr Gastroenterol Nutr 2011;52(5):585-589. 16. Simonin M, D'Agostino I, Lebreton M, Jughon O, Hamza J, Oualha M. Bilateral vocal palsy following coin cell lithium battery ingestion: a case report and review. Eur J Pediatr 2013;172(7):991-993. 17. Samad L, Ali M, Ramzi H. Button battery ingestion: hazards of esophageal impaction. J Pediatr Surg 1999;34(10):1527-1531. 18. Krom H, Visser M, Hulst JM, Wolters VM, Van den Neucker AM, de Meij T, et al. Serious complications after button battery ingestion in children. Eur J Pediatr 2018;177(7):1063-1070. 19. Samad L, Ali M, Ramzi H. Button battery ingestion: hazards of esophageal impaction. J Pediatr Surg 1999;34(10):1527-1531. 20. Litovitz T, Schmitz BF. Ingestion of cylindrical and button batteries: an analysis of 2382 cases. Pediatrics 1992;89(4 Pt 2):747-757. 21. Eisen GM, Baron TH, Dominitz JA, Faigel DO, Goldstein JL, Johanson JF, et al. Guideline for the management of ingested foreign bodies. Gastrointest Endosc 2002;55(7):802-806. 22. Kramer RE, Lerner DG, Lin T, Manfredi M, Shah M, Stephen TC, et al. Management of ingested foreign bodies in children: a clinical report of the NASPGHAN Endoscopy Committee. J Pediatr Gastroenterol Nutr 2015;60(4):562-574. 23. Litovitz T, Whitaker N, Clark L. Preventing battery ingestions: an analysis of 8648 cases. Pediatrics 2010;125(6):11781183.

\section{Tables}

Table 1 Baseline characteristics, complications and outcomes of 14 children 


\begin{tabular}{|c|c|c|c|c|c|c|c|c|c|c|c|c|c|}
\hline Site & No. & $\begin{array}{l}\text { Age } \\
\text { (month) }\end{array}$ & $\begin{array}{l}\text { Battery } \\
\text { retention } \\
\text { time (h) }\end{array}$ & Fever & Dysphagia & Salivation & Vomiting & $\begin{array}{l}\text { Abdominal } \\
\text { pain }\end{array}$ & $\begin{array}{l}\text { Diameter } \\
(\mathrm{mm})\end{array}$ & $\begin{array}{l}\text { Mucosal } \\
\text { injury }\end{array}$ & $\begin{array}{l}\text { Retention } \\
\text { time of } \\
\text { gastric } \\
\text { tube }(\mathrm{d})\end{array}$ & Complications & Outcomes \\
\hline \multirow[t]{10}{*}{1} & 1 & 17 & 52 & Yes & Yes & Yes & No & No & 22 & $\begin{array}{c}\text { E, S, } \\
\text { ME and } \\
\text { PM }\end{array}$ & 136 & TEF & Cure \\
\hline & 2 & 34 & 9 & No & Yes & Yes & Yes & No & 15 & E and $\mathrm{S}$ & 5 & EI & Cure \\
\hline & 3 & 14 & 34 & No & Yes & Yes & Yes & No & 20 & E and $\mathrm{S}$ & 12 & EI & Cure \\
\hline & 4 & 22 & 17 & No & Yes & No & No & No & 22 & $\begin{array}{c}\text { E, S and } \\
\text { PM }\end{array}$ & 25 & EI & Cure \\
\hline & 5 & 15 & 5 & No & Yes & Yes & Yes & No & 18 & $\mathrm{H}$ and $\mathrm{S}$ & 6 & EI & Cure \\
\hline & 6 & 16 & 99 & No & Yes & No & Yes & No & 13 & $\mathrm{E}$ and $\mathrm{S}$ & 7 & EI & Cure \\
\hline & 7 & 31 & 12 & No & Yes & No & No & No & 22 & $\mathrm{H}$ and $\mathrm{S}$ & 7 & EI & Cure \\
\hline & 8 & 11 & 24 & Yes & Yes & Yes & Yes & No & 15 & $\begin{array}{c}\text { E, S and } \\
\text { PM }\end{array}$ & 30 & EI & Cure \\
\hline & 9 & 9 & 31 & No & Yes & Yes & Yes & No & 18 & $\begin{array}{c}\text { E, S and } \\
M E\end{array}$ & 16 & EI & Cure \\
\hline & 10 & 9 & 15 & No & Yes & Yes & Yes & No & 22 & $\begin{array}{c}\mathrm{E}, \mathrm{S} \text { and } \\
\mathrm{H}\end{array}$ & 11 & EI & Cure \\
\hline 2 & 11 & 29 & 76 & Yes & Yes & Yes & Yes & No & 21 & $\begin{array}{c}\text { E, S, } \\
\text { ME and } \\
\text { PM }\end{array}$ & 9 & $\mathrm{AEF}$ & Death \\
\hline \multirow[t]{2}{*}{3} & 12 & 85 & 13 & No & No & No & No & Yes & 21 & $\mathrm{E}$ and $\mathrm{S}$ & 7 & EI & Cure \\
\hline & 13 & 15 & 4 & No & Yes & Yes & No & Yes & 20 & $\mathrm{E}$ and $\mathrm{S}$ & 12 & EI & Cure \\
\hline 4 & 14 & 102 & 1 & No & No & No & No & No & 10 & None & 0 & None & Cure \\
\hline
\end{tabular}

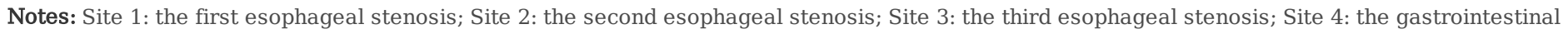
tract. E: mucosal erosion; S: mucosal swelling: ME: mucosal errhysis; H: mucosal congestion; PM: mucosal attached pseudo membrane. TEF: esophagotracheal fistula; EI: esophageal injury; AEF: aortoesophageal fistula. It should be noted that only 9 children had laryngeal stridor.

Figures
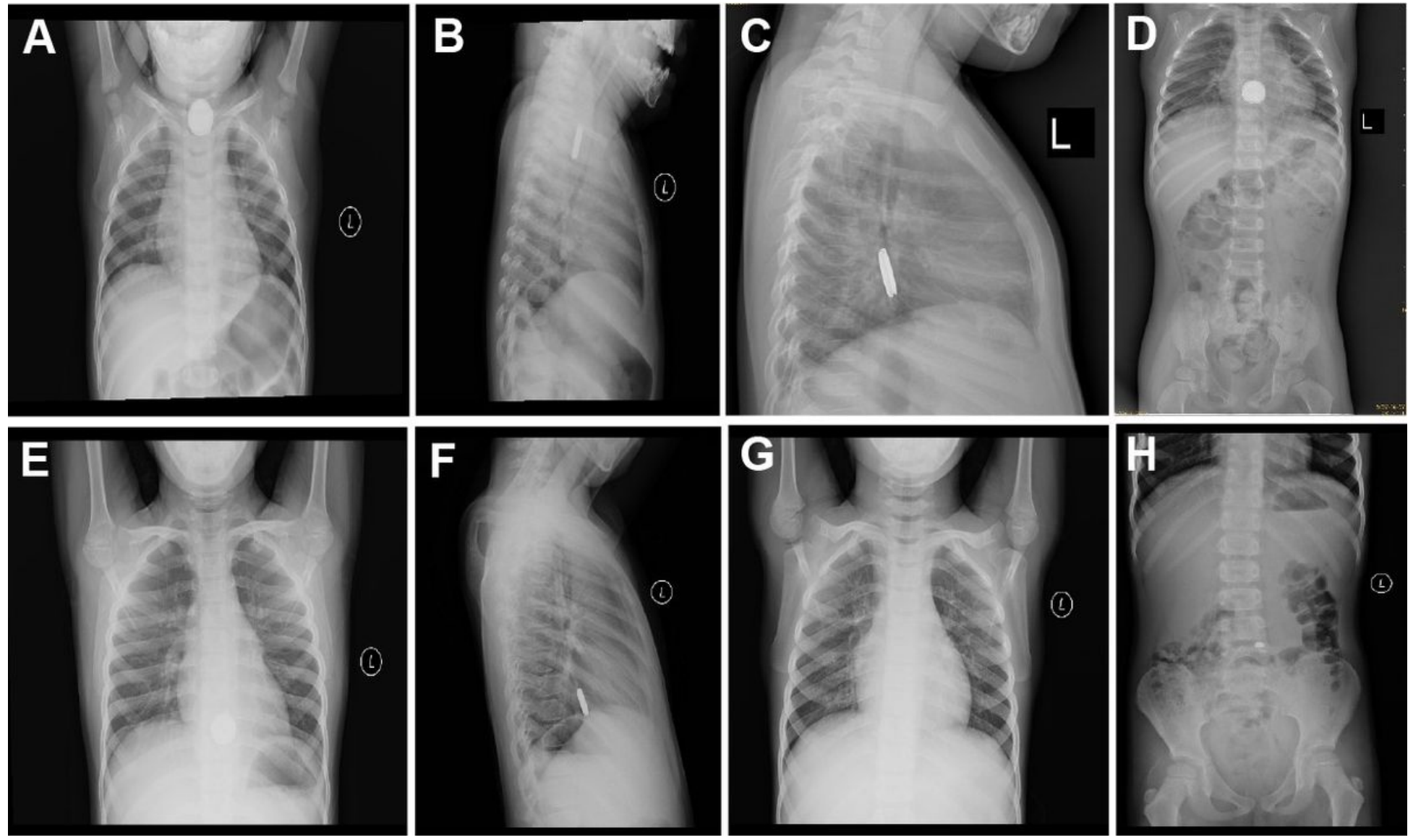

Figure 1

The button batteries lodged in the upper gastrointestinal tract Button batteries were lodged in the first (A, B), second (C, D) and third (E, F) stenoses of esophagus and in the gastrointestinal tract $(G, H)$. The child was complicated with esophagotracheal fistula 12 days after removing 
the button battery (A, B); the child was dead due to complication of aortoesophageal fistula 13 days after removing the button battery (C, D).
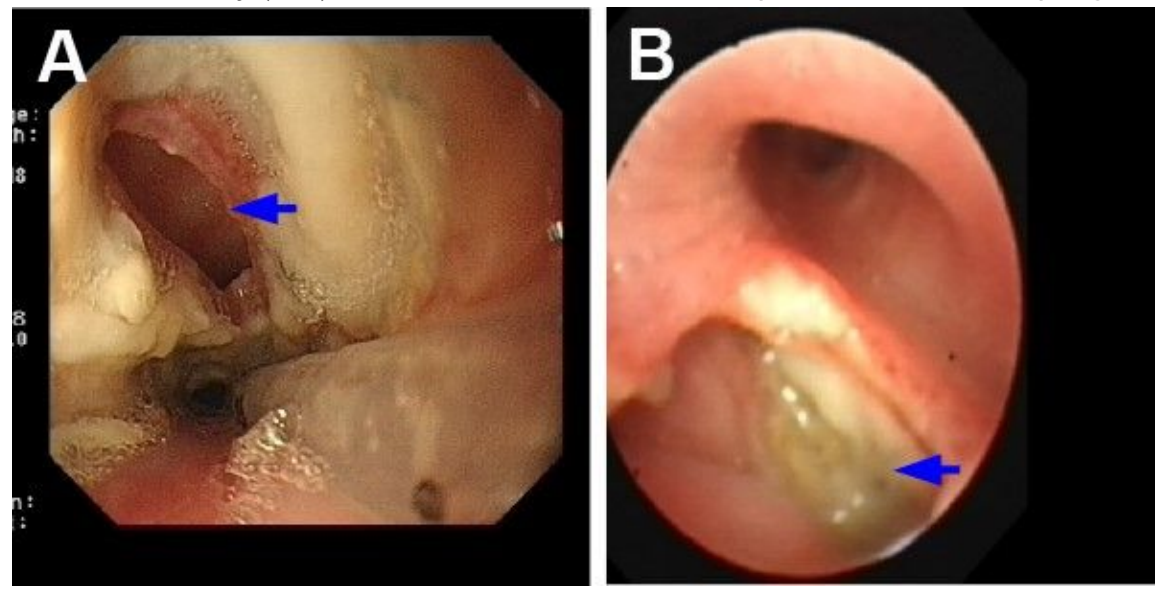

\section{Figure 2}

The child with the ingested button battery lodged in the first stenosis of esophagus was complicated with esophagotracheal fistula. (A) esophagotracheal fistula under gastroscopy; (B) tracheal fistula under fiberoptic bronchoscopy.
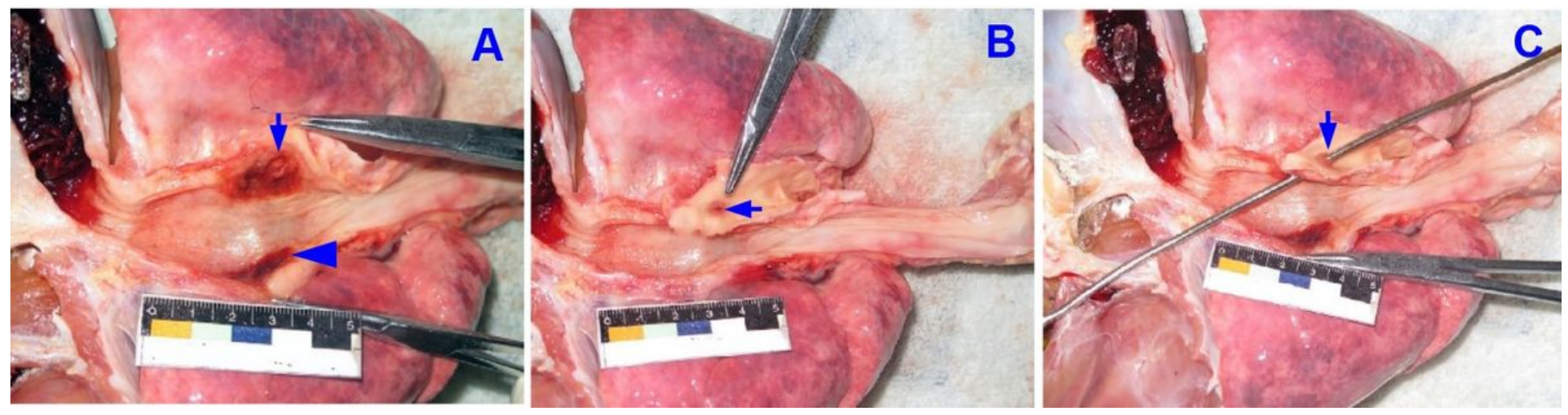

\section{Figure 3}

The autopsy result of child with ingested button battery lodged in the second stenosis of esophagus. The child died of aortoesophageal fistula. (A) ulcer area in the left (arrow) and right (arrowhead) side of esophagus; (B) the crevasse in thoracic aortic intima (arrow); (C) aortoesophageal fistula (arrow). 